\title{
The Effect of Pottery Therapy on Heart Rate Variability in College Students with Mental Health Problems
}

\author{
Nakamura $\mathbf{S}^{1}$, Sonezaki $\mathrm{S}^{2}$, Hayashida $\mathrm{Y}^{3}$ and Sato $\mathrm{T}^{4 *}$ \\ ${ }^{1}$ Graduate School of Medical Science, Saga University, Saga, Japan \\ ${ }^{2}$ Graduate School of Engineering, Kyushu Institute of Technology, Kitakyushu, Japan \\ ${ }^{3}$ Graduate School of Science and Engineering, Saga University, Saga, Japan \\ ${ }^{4}$ Health Care Center, Saga University, Saga, Japan
}

\begin{abstract}
Objective: Pottery therapy is an art therapy technique, with effects having been reported by questionnaire analysis and case studies. However, it is not precisely clear what physiological changes are caused by pottery therapy. The aim of this study was to investigate the effects of pottery therapy using heart rate variability analysis.

Methods: Participants for this study were 11 undergraduate students at Saga University in Japan who had a mental health problem. The pottery therapy consisted of a group session of up to 5 persons once a week. Each session took place for about 90 min. Heart rate variability (HRV) was measured for 5 minutes before and after the pottery therapy. Participants were divided into two groups: a therapy group and a control group. HRV was calculated for time and frequency domains using the check my heart software.
\end{abstract}

Results: In the pottery therapy group, the Poincare plot parameters SD1, SD2, S, and RMSSD were significantly increased following therapy $(p<0.05)$. There was no significant change of LF/HF between pre-test and post-test $(p=0.374)$. In the control group, no parameters were significantly changed during a session without pottery therapy.

Conclusion: The new finding from this study is that pottery therapy is effective as demonstrated by Poincaré analysis.

Keywords: College students; Heart rate variability; Poincaré plot; Pottery therapy

\section{Introduction}

In general, pottery therapy using clay media is one technique of art therapy. Clay is a familiar material in both art therapy and psychotherapy [1]. Clay is a flexible, natural material that can be built up and molded into different states of matter and textures. The processes of working with clay may stimulate all the senses, involving very primary forms of expression and communication [2].

Clay therapy may therefore provide a powerful medium to work through many core issues (e.g., anger, grief, fear) and move the client suffering from crippling emotional experiences toward flourishing opportunities for inner growth and healing [3]. Counselors use clay as a means to enhance the therapeutic relationship and support clinical processes. Clay has always been a popular toy for children and more recently a tool of play therapy. Clay provides children with a natural method of connection and expression [4]. It has been reported that low social economic status adolescents who received pottery workcentered group art therapy showed a statistically significant increase in ego resilience [5]. The use of clay as a therapeutic tool for psychiatric patients promotes creativity and self-consciousness, and was shown to benefit those seeking anxiety relief [6]. As described above, the effects of pottery therapy have typically been reported via questionnaires and case studies. However, it is not clear precisely what physiological changes are produced by pottery therapy.

In recent years, more direct approaches have been applied for certain kinds of art therapy, such as music therapy, to distinguish the mechanisms of the therapies on nervous system physiology [7-9]. In these studies, heart rate variability (HRV) has been used to gauge activation of the sympathetic and parasympathetic nervous systems [7].

Some reports indicate that art therapy impacts on the autonomic nervous system; however, few quantitative studies have been conducted to determine how pottery therapy influences the autonomic nervous system. The aim of this study was to investigate the effect of pottery therapy using HRV analysis in college students with mental problems.

\section{Method}

\section{Participants}

Participants for this study were 11 undergraduate students (5 female and 6 male) who visited the Health Care Center at Saga University, Japan. Their ages ranged from 19 to 23 years, with a mean of 20.6 years. They each had a mental health problem, and their psychiatric diagnoses were made by a designated psychiatrist (license number 8314 in Japan; one of the authors, TS) based on the Diagnostic Statistical Manual of Mental Disorders, version V [10]. Diagnoses included Major Depressive Disorder ( $\mathrm{N}=5)$, Schizophrenia $(\mathrm{N}=1)$, ObsessiveCompulsive Disorder $(\mathrm{N}=1)$, Social Anxiety Disorder $(\mathrm{N}=1)$, Autistic Spectrum Disorder $(\mathrm{N}=2)$, and Attention -Deficit/Hyperactivity Disorder $(\mathrm{N}=1)$.

\section{Procedures}

The pottery therapy included a group session of up to 5 persons

*Corresponding author: Sato T, MD PhD, Health Care Center, Saga University, 1 Honjo, Saga 840-8502, Japan, Tel: +81-952-28-8181; Fax: +81-952-28-8184; E-mail: satot@cc.saga-u.ac.jp

Received October 25, 2017; Accepted October 31, 2017; Published November 07, 2017

Citation: Nakamura S, Sonezaki S, Hayashida Y, Sato T (2017) The Effect of Pottery Therapy on Heart Rate Variability in College Students with Mental Health Problems. J Ment Disord Treat 3: 144. doi:10.4172/2471-271X.1000144

Copyright: (c) 2017 Nakamura S, et al. This is an open-access article distributed under the terms of the Creative Commons Attribution License, which permits unrestricted use, distribution, and reproduction in any medium, provided the original author and source are credited. 
once a week in the afternoon from September 2015 to March 2017. Each session took about $90 \mathrm{~min}$. These were held in the relaxation room at Saga University Health Care Center. During the group sessions, participants freely made cups, bowls, plates, and sculptures using clay by hand forming. An art therapist (SN) participated in each session, and maintained a non-judgmental attitude about their ideas and the works.

\section{Measurements}

Participants were initially seated for $5 \mathrm{~min}$ to stabilize their heart rate variability (HRV) measurements. Electrocardiograph electrode pads were affixed to the center of the inside of both arms (Figure 1). Before and after the clay-work (lasting about 90 minutes), measurements were made for 5 minutes under the same conditions. Participants were classified as belonging to the therapy group when they worked with clay, and classified into the control group when he did not work with clay. HRV was calculated for time and frequency domains using Check My Heart software ${ }^{\varpi}$ (Daily Care BioMedical, Japan).

\section{Data analysis}

Heart rate responds dynamically to physiologic perturbations mediated by the autonomic nervous system via efferent vagal and sympathetic nerve impulses.

Fluctuations in heart rate reflect autonomic modulation and have prognostic significance in pathological states [11]. Healthy cardiac activity involves a high degree of beat-to-beat variability [12], and HRV may be used to assess autonomic imbalances [13], diseases, and risk of mortality. Parasympathetic activity and HRV have been associated with a wide range of conditions including cardiovascular disease [14].

\section{Time domain measurements (Poincaré plot of R-R interval} (RRI))

The time domain HRV analysis was evaluated by calculating widely accepted Poincaré plot parameters [15]. The Poincaré plot is a scatterplot of the current RRI plotted against the preceding RRI (Figure 2) The Poincaré plot analysis is a quantitative visual technique, whereby the shape of the plot is categorized into functional classes. The plot provides summary information as well as detailed beat-to-beat information related to the behavior of the heart. Parameters analyzed included the following:

(1) SD1 - the standard deviation measuring the dispersion of points in the plot across the identity line. All points of the Poincaré plot are projected on a line perpendicular to the line of identity, and

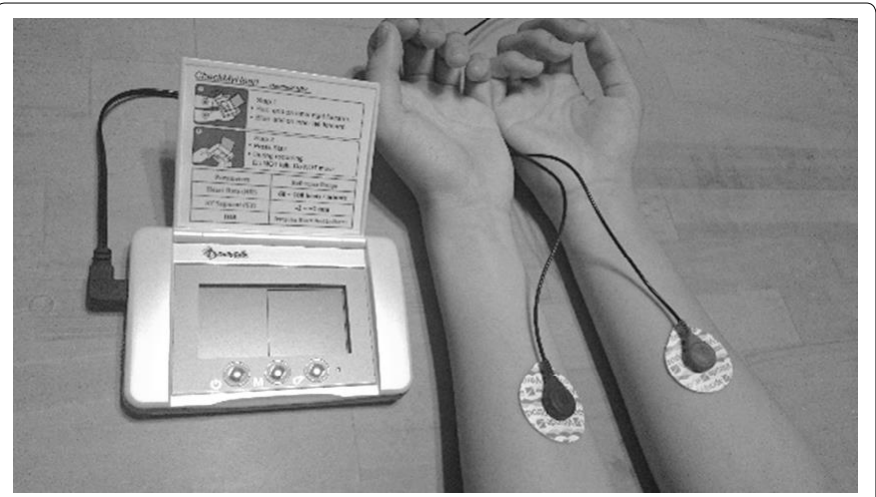

Figure 1: Measurement: Electrocardiograph electrode pads were affixed to the center of the inside of both arms.

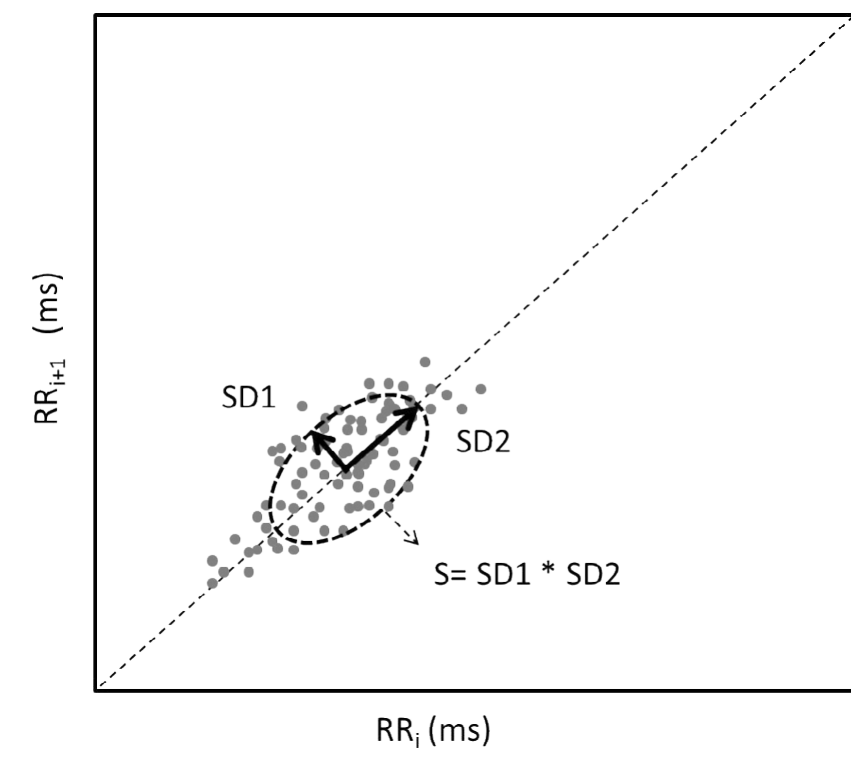

Figure 2: The Poincaré plot of heart rate recording. SD1 measures the dispersion of points across the identity line, and SD2 measures the dispersion of points along the identity line of the Poincaré plot. Both SD1 and SD2 are axes of an imaginary ellipse whose shape is visible in the diagram.

the standard deviation of the resulting distribution is calculated. This parameter is usually interpreted as a measure of short-term HRV.

(2) SD2 - the standard deviation measuring the dispersion of points along the identity line. All points are projected on the identity line, and the standard deviation is calculated. This variable is interpreted as a measure of both short- and long-term HRV.

(3) $\mathrm{S}$ - corresponds to the area of an imaginary ellipse $(\mathrm{S}=\mathrm{SD} 1 \times$ SD2) with the axes of lengths SD2 (parallel to the identity line) and SD1 (perpendicular to the line of identity).

In this study this descriptor is interpreted as a measure of total HRV because it has the qualities required for such a descriptor, e.g., it grows with the growth of either SD1 or SD2, or with both, and it remains constant if SD1 grows at the rate at which SD2 decreases [16].

\section{Other parameters from time domain measurements}

Root mean square successive difference (RMSSD) - The most common variables calculated as differences between normal RRI are RMSSD. It is the square root of the successive differences between normal $\mathrm{R}$ waves (NN intervals), essentially the average change in interval between beats [11]. The RMSSD is considered to reflect cardiac parasympathetic activity, especially the Poincaré plot diameter SD1, a measure of instantaneous beat-to-beat variability. The Poincaré plot dimension SD1 and the time domain measure RMSSD are both mathematically simple, unambiguous measures of HRV [17].

\section{Frequency domain measurement (spectrum density analysis)}

Spectrum density analysis is the process of determining the frequency domain representation of a time domain signal, and most commonly employs the Fourier transform. The Fast Fourier Transform (FFT) is an efficient method for measuring autonomic nerve function. The power spectrum is divided into two main frequency ranges: low-frequency power (LF) (from 0.04 to $0.15 \mathrm{~Hz}$ ), high-frequency power (HF) (from 0.15 to $0.4 \mathrm{~Hz}$ ), and the ratio LF/HF. HF is usually interpreted as reflecting high-frequency oscillations caused mainly by 
Citation: Nakamura S, Sonezaki S, Hayashida Y, Sato T (2017) The Effect of Pottery Therapy on Heart Rate Variability in College Students with Mental Health Problems. J Ment Disord Treat 3: 144. doi:10.4172/2471-271X.1000144

Page 3 of 5

changes in vagal tone, which are responsible for short-term HRV. LF describes low-frequency oscillations that depend on both sympathetic and parasympathetic activities and are responsible for long-term HRV. The ratio of LF to HF (LF/HF) reflects the balance between low- and high-frequency oscillations, i.e., long- and short-term HRV [16].

\section{Ethical considerations}

We received approval for this research from the Saga Medical School Ethics Committee. In addition, we carefully explained the purpose of this study to participants, and obtained informed consent orally.

\section{Statistics analysis}

The data were analyzed using the statistical software SPSS (version 21.0). The level of significance was set at 0.05 for all tests. The Wilcoxon one sample (matched pairs) signed rank test was used to verify a change from pre-test to post-test in each session.

\section{Results}

\section{The effect of pottery therapy on HRV}

Comparison of the various values and $p$-values associated with differences between pre-test and post-test are summarized in Table 1. In the therapy group, the Poincaré plot parameters SD1, SD2, S, and RMSSD were significantly increased after pottery therapy $(\mathrm{p}<0.05)$. However, there was no significant change in LF/HF between pre-test and post-test $(\mathrm{p}=0.374)$. Regarding the control group, no parameters were significantly changed during the session without pottery therapy. These data clearly indicate that pottery therapy has a significant effect on HRV. Two individual cases are described below.

\section{Case 1}

'Mari' was a 20-year-old female suffering from schizophrenia since high school. She was interested in making something with clay.

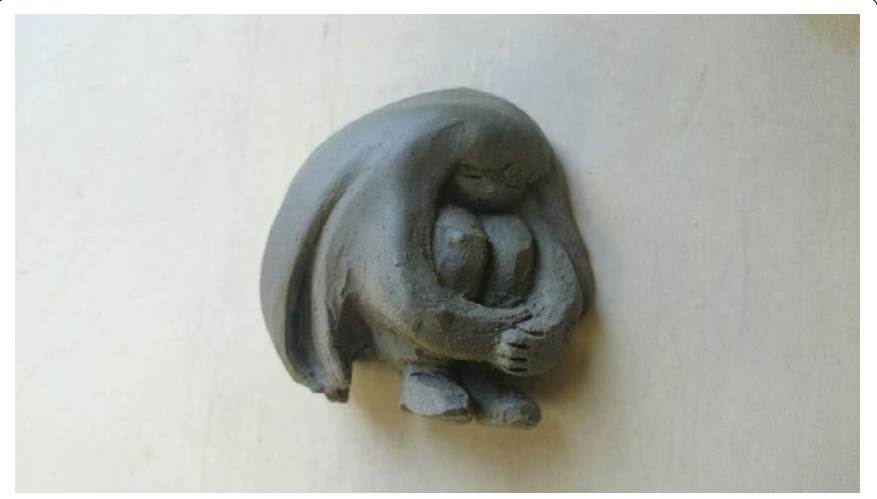

Figure 3: A sculpture of a little girl by Case 1
"Actually, I witnessed a scene where people were arguing angrily this morning, so I became emotionally distressed, feeling a tightness in my chest."

She made a sculptural relief of a little girl who holds herself, and calmed down during the pottery therapy (Figure 3). "I' want to place what I made in a favorite place in my room as a decoration".

"I like the sense of touch, weight, and coolness of the clay" shows the Poincaré plot between the pre-test and post-test during this session (Figure 4).

\section{Case 2}

'Ko-ji' was a 22-year-old male who had a social anxiety disorder. In particular, he could not speak to people who he just met. He was always very visibly nervous, so he wanted to overcome this through participating in the session.

He hardly talked to others, but seemed to be comfortable to be in the group session. He did not have to talk with anyone while he concentrated on creating work. The therapist asked him, "How are you feeling today?". He replied in a small voice, "Better than before." (Figure 5) shows the work he created. (Figure 6) shows the Poincaré plot between the pre-test and post-test during this session.

In both cases, the distribution of the plot area of Poincaré was more scattered after the therapy.

\section{Discussion}

HRV alterations have been proposed as biomarkers for disease susceptibility. It may be possible to use HRV to assess autonomic imbalances, diseases, and mortality risk [14]. There is growing evidence for the role of the autonomic nervous system (ANS) in a wide range of diseases [18]. HRV parameters assess the balance of sympathetic and parasympathetic influences on heart rate [19]. Analysis of HRV based on short-term analysis of the Poincaré plot provides a sensitive, non-invasive measurement of autonomic input to the heart [20]. The Poincaré plot is more visually discernible in the detection of autonomic changes, including the potential to evaluate dynamic changes [21].

Poincaré plots that reflect extremely low HRV have been classified as abnormal. SD 1 and 2 are determined by fitting an ellipse to the Poincaré plot. SD 1 is the short axis of this ellipse and SD 2 is the long axis. This measure has proved useful for detecting editing problems that significantly influence the calculation of HRV variables [11]. The RMSSD is sensitive to high-frequency heart period fluctuations in the respiratory frequency range, and has been used as an index of vagal cardiac control. The RMSSD measure correlates well with frequency domain indices of high-frequency HRV [22]. High frequency HRV represents primarily parasympathetic influences [23]. Using Poincaré parameters, we determined that the area of the plot after pottery therapy was significantly expanded.

\begin{tabular}{|c|c|c|c|c|c|c|c|}
\hline & \multicolumn{3}{|c|}{ Control } & & \multicolumn{3}{|c|}{ Pottery Therapy } \\
\hline & Pretest & Posttest & $P$ & & Pretest & Posttest & $p$ \\
\hline SD1 & $18.6 \pm 11.5$ & $18.3 \pm 11.1$ & 0.657 & SD1 & $14.6 \pm 9.30$ & $20.9 \pm 13.4$ & $0.013^{*}$ \\
\hline SD2 & $56.2 \pm 30.4$ & $52.9 \pm 26.9$ & 0.182 & SD2 & $47.2 \pm 30.2$ & $66.3 \pm 40.8$ & $0.006^{*}$ \\
\hline $\mathrm{S}$ & $1343 \pm 1369$ & $1225 \pm 1239$ & 0.929 & $S$ & $914 \pm 961$ & $1916 \pm 1649$ & $0.041^{*}$ \\
\hline RMSSD & $26.3 \pm 16.2$ & $25.9 \pm 15.7$ & 0.657 & RMSSD & $20.7 \pm 13.2$ & $29.5 \pm 18.9$ & $0.013^{*}$ \\
\hline LF/HF & $2.55 \pm 2.09$ & $2.97 \pm 2.7$ & 0.477 & LF/HF & $3.75 \pm 2.84$ & $2.78 \pm 0.98$ & 0.374 \\
\hline
\end{tabular}

Data are mean \pm SE (standard error) of each value. $p$ values: posttest vs. pretest by Wilcoxon one sample (matched pairs) signed rank test.

Table 1: Measures of various heart rate variability indices pre- and post-therapy. 
Citation: Nakamura S, Sonezaki S, Hayashida Y, Sato T (2017) The Effect of Pottery Therapy on Heart Rate Variability in College Students with Mental Health Problems. J Ment Disord Treat 3: 144. doi:10.4172/2471-271X.1000144
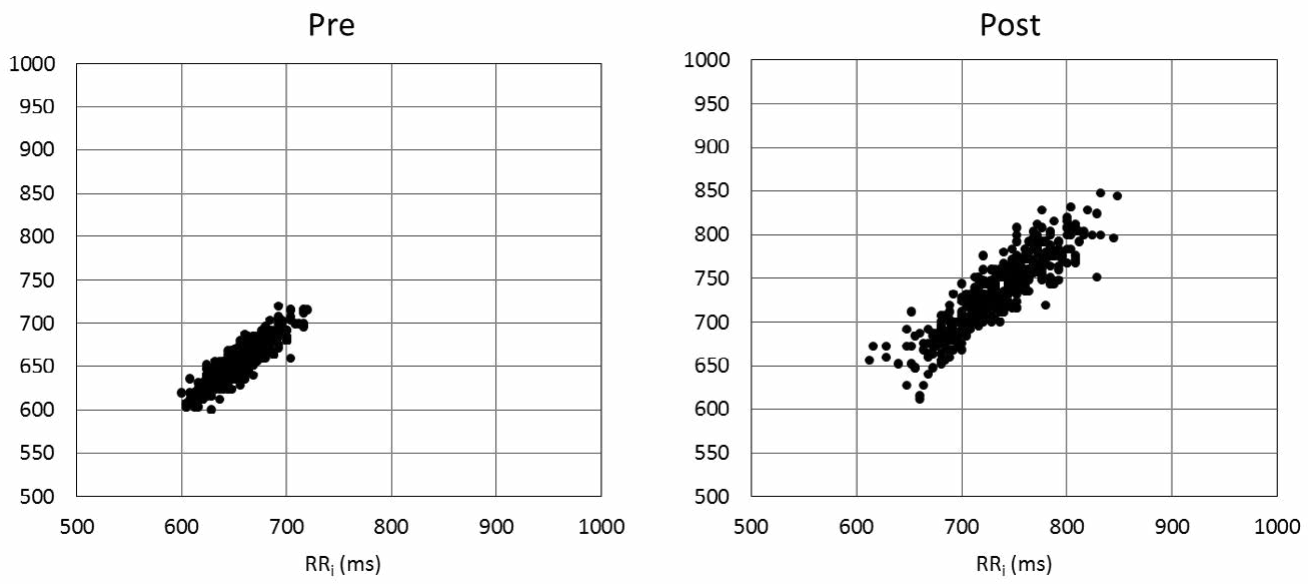

Figure 4: The Poincaré plot of HRV, pre and post-test (Case 1)

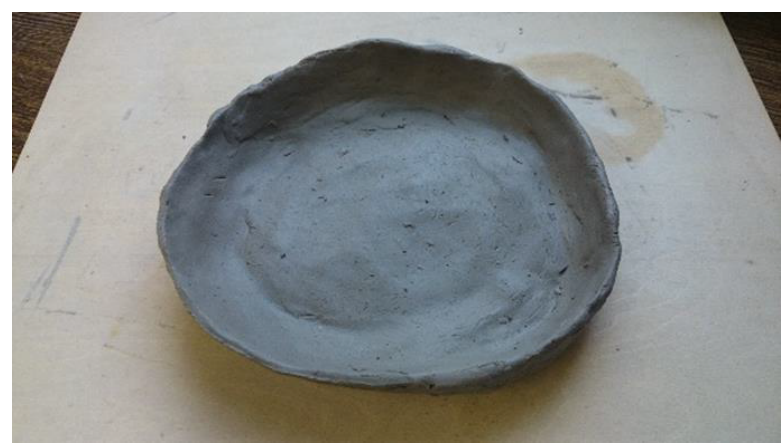

Figure 5: A plate work by Case 2 .
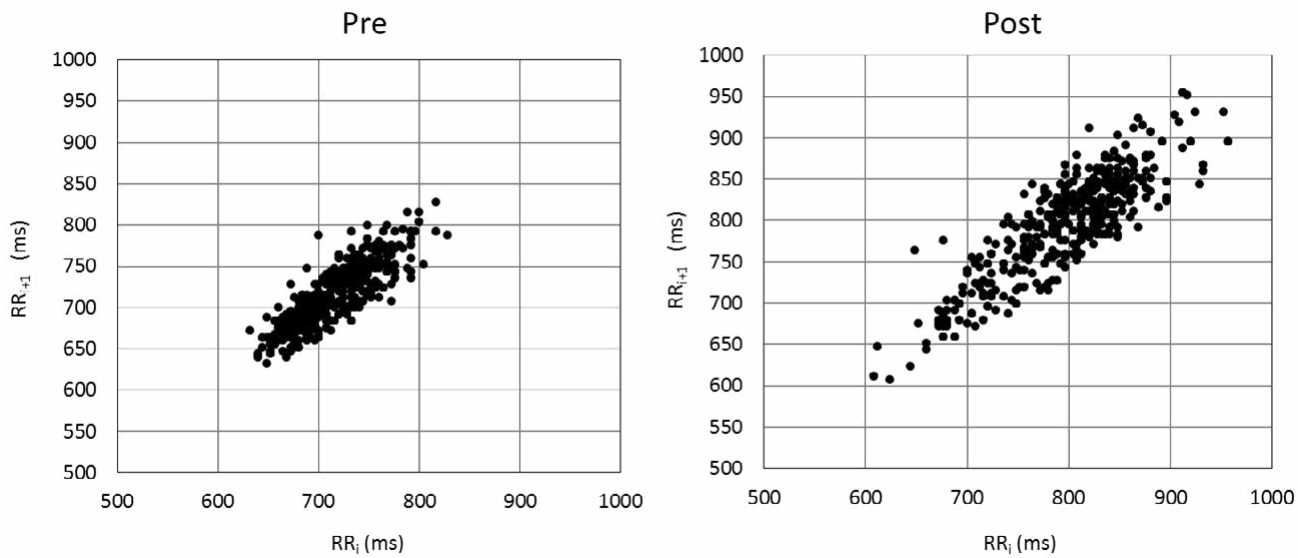

Figure 6: The Poincaré plot of HRV, pre and post-test (Case 2).

The results obtained this study show that pottery therapy has positive effects on the following HRV parameters: SD1, SD2, S, and RMSSD. These parameters were significantly increased after pottery therapy. The distribution of the plot of Poincaré exhibited a significantly larger spread, indicating that pottery therapy may stabilize the ANS.

Comparatively, there was no significant difference in $\mathrm{LF} / \mathrm{HF}$ between pre- and post-therapy tests. This result may be due to individual differences, including variables related to environment, as well as physical and mental status. Billman [24] reported that LF/ HF values may not reflect mental and physical stabilities. He also emphasized that LF/HF values could not accurately quantify cardiac sympatho-vagal balance either in health or disease. From the viewpoint of this study, therefore, LF/HF values might not be a suitable indicator that reflects the beneficial effects of pottery therapy. 
The effect of pottery therapy also has been evaluated via questionnaires and case studies. According to mean BHS scores, it was found that hopelessness scores decreased after clay therapy in patients with neurological disorders [25]. Clay therapy and narrative therapy reduce anxiety symptoms in children with anxiety disorders as measured by the Child Symptom Inventory-4: Parent Checklist [26]. The characteristics of the clay and its ease of use created a sense of security and self-efficacy. Many participants felt that working with clay enabled relief and a release, and also created a sense of calm [2]. The transformative nature of clay work was an instigator for mentalization, and reflected the existence of different options for self and relationships (that the self can establish), as well as the inner power to choose and to change, and/or to be changed [27]. Clay's three dimensionalities provided the opportunity to see the sculptures from more than one vantage point [28], and thus enabled an observation of multiple, and sometimes hidden, aspects in the complex subjective experience of being a mother [29].

As described above, the positive effect of pottery therapy using clay has been reported; however; it is not clear precisely what physiological changes are caused by pottery therapy. In this study, we were able to demonstrate its effect for the first time in a more scientific way using HRV, and our data indicates that pottery therapy promotes the stabilization of autonomic nervous system function.

The pottery therapy in this study seemed to be effective within the following factors: 1) participants were interested in making something with clay, 2) there was no discomfort in touching clay, 3) while in some cases, they were not good at verbal communication, they were able to relax while creating work requiring concentration, 4) they were satisfied with their work, and 5) from the form or expression that appears during working, they were aware of their own projected wishes and encountered unexpected self-consciousness.

\section{Limitations}

However, limitations of this study include the small number of participants. We are not yet able to conclude that pottery therapy is effective for individuals with mental health problems. Further investigation will be needed to clarify additional physiological effects of pottery therapy beyond HRV measurement.

\section{Acknowledgments}

We are grateful to the college students who participated in this research. We also thank the staff of the Health Care Center at Saga University.

\section{References}

1. Sholt M, Gavron T (2006) Therapeutic Qualities of Clay-work in Art Therapy and Psychotherapy: A Review. Art Therapy Journal of the American Art Therapy Association 23: 66-72.

2. Snir S, Regev D (2013) A dialog with five art materials: Creators share their art making experiences. The Arts in Psychotherapy 40: 94-100.

3. Sherwood P (2004) The Healing Art of Clay Therapy. Melbourne, Australian Council for Educational Research Ltd.

4. Schaefer CE, Kaduson HG (2006) Contemporary Play Therapy. New York. The Guilford Press.

5. Jang H, Choi S (2012) Increasing ego-resilience using clay with low SES (Socia Economic Status) adolescents in group art therapy. The Arts in Psychotherapy 39: $245-250$.

6. Morais AH, Roecker S, Salvagioni DAJ, Eler GJ (2013) Significance of clay psychiatric patients admitted in a day hospital. Investigación y Educación en Enfermería 32: 128-138.

7. Iwanaga M, Kobayashi A, Kawasaki C (2005) Heart rate variability with repetitive exposure to music. Biological Psychology 70: 61-66.
8. Etzel JA, Johnsen EL, Dickerson J, Tranel D, Adolphs R (2006) Cardiovascular and respiratory responses during musical mood induction. International Journal of Psychophysiology 61: 57-69.

9. Chuang CY, Han WR, Li PC, Young ST (2010) Effects of music therapy on subjective sensations and heart rate variability in treated cancer survivors: $A$ pilot study. Complementary Therapies in Medicine 18: 224-226.

10. American Psychiatric Association (2013) Diagnostic and Statistical Manual of Mental Disorders, Fifth Edition (DSM-V). Washington, DC. American Psychiatric Association.

11. Kleiger RE, Stein PK, Bigger Jr.JT (2005) Heart Rate Variability: Measuremen and Clinical Utility. Autonomic Nervous System 10 (1): 88-101.

12. Kemp AH, Quintana DS, Gray MA, Felmingham KL, Brown K, et al. (2010) Impact of Depression and Antidepressant Treatment on Heart Rate Variability: A Review and Meta-analysis. Biological Psychiatry 67: 1067-1074.

13. Falcone C, Colonna A, Bozzini S, Matrone B, Guasti L, et al. (2014) Cardiovascular Risk Factors and Sympatho-vagal Balance: Importance of Time-domain Heart Rate Variability. Clinical \& Experimental Cardiology 5: 289.

14. Thayer JF, Yamamoto SS, Brosschot JF (2009) The relationship of autonomic imbalance, heart rate variability and cardiovascular disease risk factors. International Journal of Cardiology.

15. Task Force of the European Society of Cardiology and the North American Society for Pacing and Electrophysiology (1996) Heart Rate Variability: Standards of Measurement, Physiological Interpretation, and Clinical Use. Circulation 93: 1043-1065.

16. Guzik P, Piskorski J, Krauze T, Schneider R, Wesseling KH, et al. (2007) Correlations between the Poincaré Plot and Conventional Heart Rate Variability Parameters Assessed during Paced Breathing. The Journal of Physiological Sciences 57(1): 63-71.

17. Penttilä J, Helminen A, Jartti T, Kuusela T, Huikuri HV, et al. (2001) Time domain, geometrical and frequency domain analysis of cardiac vagal outflow: effects of various respiratory patterns. Clinical Physiology 21: 365-376.

18. Thayer JF, Lane RD (2007) The role of vagal function in the risk for cardiovascular disease and mortality. Biological Psychology 74: 224-242.

19. Nagpal ML, Gleichauf K, Ginsberg JP (2013) Meta-Analysis of Heart Rat Variability as a Psychophysiological Indicator of Posttraumatic Stress Disorder. Trauma \& Treatment 3: 182.

20. Kamen PW, Krum H, Tonkin AM (1996) Poincaré plot of heart rate variability allows quantitative display of parasympathetic nervous activity in humans. Clinical Science 91: 201-208.

21. Hsu CH, Tsai MY, Huang GS, Lin TC, Chen K-P, et al. (2012) Poincaré plot indexes of heart rate variability detect dynamic autonomic modulation during general anesthesia induction. Acta Anaesthesiologica Taiwanica 50: 12-18.

22. Berntson GG, Lozano DL, Chen Y-J (2005) Filter properties of root mean square successive difference (RMSSD) for heart rate. Psychophysiology 42 246-252.

23. Thayer JF, Åhs F, Fredrikson M, Sollers III JJ, Wager TD (2012) A meta analysis of heart rate variability and neuroimaging studies: Implications for heart rate variability as a marker of stress and health. Neuroscience and Biobehavioral Reviews 36: 747-756.

24. Billman GE (2013) The LF/HF ratio does not accurately measure cardiac sympathy vagal balance. Frontiers in Physiology.

25. Akhan LU, Kurtuncu M, Celik S (2015) The Effect of Art Therapy with Clay on Hopelessness Levels Among Neurology Patients. Rehabilitation Nursing 0: 1-8.

26. Gadow KD, Sprafkin J (1997) Child Symptom Inventory -4: screening and norms manual. Stony Brook, NY. Checkmate Plus.

27. Or MB (2010) Clay sculpting of mother and child figures encourages mentalization. The Arts in Psychotherapy 37: 319-327.

28. Buchalter SI (2004) A practical art therapy. London, Jessica Kingsley Publishers.

29. Or MB (2012) Non-verbal representations of maternal holding of preschoolers The Arts in Psychotherapy 39: 117-125. 